\title{
Intelligent Impairment Aware Routing Algorithm for All-Optical Networks based on Ant Colony Optimization Trained by Particle Swarm Optimization
}

\author{
Carmelo J. A. Bastos-Filho, Renato M. Fernandes, Danilo F. Carvalho, \\ George M. Cavalcanti Junior, Daniel A. R. Chaves and, Joaquim F. Martins-Filho
}

\begin{abstract}
In this paper, we propose and analyze the performance of an adaptative impairment aware routing algorithm (IA-RWA) for all-optical networks based on a bio-inspired optimization technique, called Ant Colony Optimization (ACO). We propose a novel heuristic to be used by the routing algorithm combining the physical distance and the wavelengths availability of the optical links. These two metrics are weighted in a training stage. The algorithm was trained by another optimization technique based on swarm collective intelligence, called Particle Swarm Optimization (PSO). We show that our IA-RWA algorithm can learn during the training stage and adapts itself to the network conditions, performing equally or even better than the classical routing algorithms.
\end{abstract}

Keywords-RWA algorithms, All-Optical Networks, Ant Colony Optimization, Particle Swarm Optimization.

\section{INTRODUCTION}

The ever increasing demand on Internet data traffic has been the driving force for the recent developments in the telecommunication industry. The deployment of optical fibers by using wavelength division multiplexing (WDM) has been considered as the most suitable and reliable technology for long haul communication systems. Furthermore, the development of optical amplifiers and other devices such as optical add/drop multiplexers (OADMs) and optical cross-connects (OXCs) have enabled higher capacity optical links and network-level abilities [1] [2] [3].

Optical networks can be either opaque, translucent or transparent (all-optical). In opaque networks every node regenerates the signal (i.e. conversion from optical domain to electrical domain and back to optical domain) to accomplish its functions. These networks have high costs due to the wavelength transponders and electronic circuitry. In opaque networks, as the regeneration process occurs at each intermediate node, the accumulation of optical signal to noise ratio (OSNR) degradation due to physical impairments is much less important. On the other hand, in transparent networks there is no regeneration along the lightpath, i.e., the signal remains in the optical domain from source to destination nodes. Since the network

Carmelo J. A. Bastos-Filho, Renato M. Fernandes, Danilo F. Carvalho and George M. Cavalcanti Junior are with University of Pernambuco, Recife, Brazil, e-mail: cjabf@dsc.upe.br. Daniel A. R. Chaves and Joaquim F. Martins-Filho are with Federal University of Pernambuco, Recife, Brazil, email: jfmf@ufpe.br. This work is supported by FACEPE and CNPq. is bit-rate, protocol and format independent, it is easier to upgrade and it can support different types of traffic, being more suitable for modern demands. However, control issues must be included in the optical layer of the network to accomplish the switching of lightpaths and other functions with the proper Quality of Service (QoS). Moreover, these automatic switched optical networks (ASON) suffer from OSNR degradation due to the physical impairments, since the signal remains in the optical domain, accumulating noise as it propagates through several devices (fiber segments, EDFAs, OADMs, OXCs, MUX, DEMUX, etc.) [1][2][3]. In translucent networks, there are some islands of transparency.

One of the major issues that can affect the transmission performance in all-optical networks is the routing and wavelength assignment (RWA) algorithm. The dynamic RWA problem involves algorithms for efficient route selection and wavelength assignment, including signaling mechanisms to request and establish lightpaths between source and destination nodes, and upgrade the network status when the connections are established and terminated, targeting at a minimum call blocking probability [4][5]. Several papers about optical routing algorithms assume that all routes have adequate signal quality. This is not always the case, especially for large and heavily loaded networks. Although every point-to-point link in a network is designed to provide an adequate signal quality at its output, the dynamics of the network imposes drastic changes in optical parameters over the time. Classical routing algorithms based on distance (shortest path), hop count and least resistance weigth (LRW) do not take into account the transmission impairments.

Some papers have proposed to use computational intelligence techniques to solve the routing problem in communications networks using Hopfield Neural Networks [6], Genetic Algorithms [7], Particle Swarm Optimization [8] and Ant Colony Optimization [9].

Recently, some papers have proposed to solve the RWA problem in all-optical networks using Ant Colony Optimization [10][11][12][13], which will be commented in Section II.

In this paper, we propose and analyze the performance of an adaptive impairment aware routing algorithm (IA-RWA) for transparent optical networks based on Ant Colony Optimization (ACO). We propose a novel heuristic to be considered by the ants for the route calculation. It is based on optical links 
physical distance and the optical link wavelengths availability. The algorithm weights these physical impairments in a trainning stage using another optimization technique called Particle Swarm Optimization (PSO). We compare the performance of our proposal to the ACO approach based on distance applied for routing in communications networks and to other networklayer routing algorithms. We show that our IA-RWA algorithm can learn during the training stage and adapts itself to the network conditions, performing equally or even better than the classical routing algorithms, in terms of blocking probability.

This paper is structured as follows. Section II describes the routing algorithm based on ACO and its parameters. Section III presents the PSO algorithm used to obtain the best set of parameters used by the routing algorithm. Section IV presents the physical layer model and the experimental setup used in the simulations. Section $\mathrm{V}$ presents the results. In Section VI we give our conclusions.

\section{IA-RWA ALgORITHM BASED ON ANT COLONY OPTIMIZATION}

Although an ant is a simple creature, collectively ants can present a useful behavior for performing tasks such as discovering the shortest path between a food source and the nest [9]. The colony shares information through Stigmergy, that is a form of indirect communication used by ants in nature by laying a chemical substance called pheromone. The pheromone induces changes in the environment, which can be sensed by other ants.

An artificial ant can be implemented as a simple procedure that simulates the laying and sensing of pheromone. Each ant is originated in the source node $s$ and explores the network trying to find the destination node $d$. Basically, the ants are guided by the pheromone and should have a predefined lifetime $\left(T_{\text {life }}\right)$ to find their destination. $T_{\text {life }}$ quantify how long an ant remains moving along the network. An ant needs to be able to find the end of the path until $T_{\text {life }}$ is reached. This preset lifetime helps to avoid loops. When the ant finds the destination node, it returns to the source node in the reverse direction by adding pheromone to the link. In this paper the pheromone deposited on the routing tables by an ant along the nodes included in the ligthpath is unitary. Furthermore, the colony consists of a data structure that generates ants and records the nodes that they pass. In our approach we periodically generate a group of $n_{\text {ants }}$ ants with a frequency $f$ and the simulation has a total duration of $T_{\text {total }}$. After $T_{t o t a l}$, the pheromone table state indicates the best route.

However, this operation alone can lead to a stagnation process. One of the approaches used to mitigate stagnation is to configure ants so that they do not solely rely on sensing pheromone. For this purpose, one can set a mixed probability function for an ant to decide between different links. This can be done using both pheromone concentration and a heuristic function [14]. For example, an ant selects a link probabilistically using a composition function of the cost of the link and the pheromone left by previous ants. One common approach to this function is presented in equation 1 , where $P_{i j}$ is the weight used by the ant to choose the next node to visit, $P h_{i j}$ is the pheromone quantity in the link $i j, d_{i j}$ is the physical length of the link $i j$ and $d_{i j}^{\prime}$ is the normalized length of the link $i j$ [9]. In this case, the ants choose the path that presents the higher $P_{i j}$.

$$
P_{i j}=\operatorname{Rand}() \frac{P h_{i j}^{\alpha}}{d_{i j}^{\prime}},
$$

where $\alpha$ and $\beta$ are constants that weight the pheromone and the network parameter used as cost function. $\operatorname{Rand}()$ is a random number generated by a uniform distribution in the interval $[0,1]$.

We also used a technique called evaporation, where in each iteration the pheromone values at all the routing tables are reduced by a predefined factor $\delta$. This parameter describes the ratio of pheromone that evaporates in a link per iteration. $\delta$ is used to prevent pheromone concentration in optimal paths from being excessively high. A high concentration of pheromone in some links can excessively polarize the ants. This can affect the ability to explore other routes in the case of network failures. Pavani and Waldman [13] used other intersting technique to perform this target by reinforcing the paths found by the ants.

Some efforts have been made to solve the RWA problem using Ant Colony Optimization. Ngo et al. [10] adapted the algorithm to WDM optical networks. Pavani and Waldman [11] proposed a heuristic to induce the ants based on the difference between the maximum allowed power on the link and the total active power on the link. In other paper, Pavani and Waldman [12] proposed two different distributed strategies for provisioning lightpaths in the presence of optical physical-layer impairments in GMPLS networks. Pavani et al. [13] also showed that ACO can be used for restoration in wavelength-routed optical networks. In [13], a heuristic based on the link wavelength availability of the network links $\left(\Delta \lambda_{i j}\right)$ is introduced. However, they do not consider this metric simultaneously with the distance to induce the ants.

The link wavelength availability of the network links $\left(\Delta \lambda_{i j}\right)$ is defined as:

$$
\Delta \lambda_{i j}=\frac{\lambda_{i j}^{\text {available }}}{\lambda_{i j}^{\text {total }}},
$$

where $\lambda_{i j}^{\text {available }}$ is the number of available wavelengths in the link $i j$ and $\lambda_{i j}^{\text {total }}$ is the total number of wavelengths in the link $i j$.

In this paper we propose to combine the heuristics presented in [9] and [13]. The equation used to guide the ants is given by 3 .

$$
P_{i j}=\operatorname{Rand}() \frac{P h_{i j}^{\alpha} \Delta \lambda_{i j}^{\gamma}}{d_{i j}^{\beta}} .
$$

where $\Delta \lambda_{i j}$ is the link wavelength availability of the network link $i j$.

\section{PSO FOR TRAINING THE RWA ALGORITHM BASED ON} ACO

In order to find the best parameters $\alpha, \beta$ and $\gamma$, we used another optimization technique called Particle Swarm 
Optimization (PSO). We call this process as the training phase, since the IA-RWA algorithm runs several times for different values of the parameters, to find their best values. This process occurs offline, which means that it occurs before the network operation. The online routing processes occurs with the use of ACO and equation 3, with the parameters found by PSO. Martins-Filho et al [8] have also used PSO to find the best parameters for a cost function of a IRWA algorithm. However, their IA-RWA algorithm does not use ACO to find the routes.

Particle Swarm Optimization is a bio-inspired technique proposed by Kennedy and Eberhart in 1995. We used the PSO recommended by Bratton and Kennedy [15], following the pseudo-code proposed therein. The search space dimensions number was defined according to the used equation. Particles velocities were updated using the constriction factor approach [16], to avoid overflight and to improve performance. We used $k$ equal to 1 , acceleration constants $\left(c_{1}\right.$ and $\left.c_{2}\right)$ equals to 2.05 for both, observing the constriction factor condition of $\Phi>4$, for overflight free operation. The values for these parameters were previously studied in [16] and guarantee stable operation.

We used the $L_{\text {best }}$ swarm model with ring topology [15] because this is the recommended model for multimodal problems. 21 particles were used in all simulations. This swarm size respects the standard defined by Bratton and Kennedy [15].

For our simulations we observed that 150 iterations are sufficient for the algorithm to converge. This can be justified by the fact that the search space is not too large. Therefore, this number of iterations was established as the training phase period. Particles do RWA simulations as described in section IV. For each scenario, we repeat five times the training process. The blocking probabilities were calculated as the average of the simulation results.

Each ant has the lifetime configured to $T_{\text {life }}=2 \mathrm{~N}$, where $N$ is the number of network nodes. The influence of the parameter $T_{\text {life }}$ was widely studied in [17]. The value $T_{\text {life }}=2 N$ is the minimal value to guarantee that the colony has sufficient time to find optimal solutions with low error rates.

Colonies have 50 ants and new generations occurr in each iteration (i.e. $f=1$ ) with a maximum of five ants (i.e. $n_{\text {ants }}=$ 5 ), until the colony is full-filled. After finding a route, each ant returns to its origin incrementing pheromone quantity in one unit. The best value for $\delta$ found in preliminar simulations was 0.67 , and we used this in all simulations.

The training network load was defined as the one to achieve a blocking probability equal to $1 \%$ when the shortest path algorithm is adopted.

One should note that tha parameter $\alpha, \beta$ and $\gamma$ are internal parameters obtained during the trainning stage. It means that they are not defined by a network designer or operator.

\section{Physical Layer Model and Experimental Setup}

Our simulation algorithm works as follows: upon a call request it determines a route using the selected routing algorithm. Then, it selects an available wavelength from a list using the first fit algorithm. The lightpath $O S N R$ is evaluated. If it is above the pre-determined level $\left(O S N R_{T h}\right)$ the call is established. Our algorithm blocks a call if there is no available wavelength or if the $O S N R$ for the respective wavelength is below the $O S N R_{T h}$. The algorithm also evaluates the pulse broadening and checks if it is above a predefined value. If this occurs, the call is blocked. The blocked calls are lost. The blocking probability is obtained from the ratio of the number of blocked calls and the number of requested calls. For each network simulation, a set of at least $10^{5}$ calls are generated choosing randomly the source-destination pairs. We used $10^{6}$ calls when the blocking probability is around $10^{-5}$. The call request process is characterized as a Poisson process and the time duration for each established call is characterized as an exponential process. We assume circuit-switched bidirectional connections in two fibers and no wavelength conversion capabilities.

The links have the following elements: transmitter, optical switch, multiplexer, booster amplifier, optical fiber, preamplifier, demultiplexer, optical switch and receiver. In this paper we consider the following effects as physical impairments: amplifier gain saturation, ASE emission, crosstalk in the switch and polarization mode dispersion. More details about the physical layer model can be found in [18].

Figure 1 shows the network topologies used in our simulations. The first one is a regular and symmetric topology. The second one reproduces the Finland topology for long haul communication. The amplifier gains are set to compensate for the total link losses and the default parameters used in our simulations are shown in Table I.

TABLE I

DEFAULT SIMULATION PARAMETERS

\begin{tabular}{|c|c|l|}
\hline Parameter & Value & \multicolumn{1}{|c|}{ Definition } \\
\hline \hline$P_{\text {Laser }}$ & $-3 \mathrm{dBm}$ & Transmitter output power \\
\hline$P_{\text {sat }}$ & $19 \mathrm{dBm}$ & Amplifier output saturation power. \\
\hline OSNR $_{\text {in }}$ & $40 \mathrm{~dB}$ & $\begin{array}{l}\text { Optical Signal to Noise Ratio at the } \\
\text { transmitter. }\end{array}$ \\
\hline OSNR $_{\mathrm{QoS}}$ & $23 \mathrm{~dB}$ & $\begin{array}{l}\text { Minimum Optical Signal to Noise } \\
\text { Ratio at the receiver. }\end{array}$ \\
\hline$B$ & $40 \mathrm{Gbps}$ & Transmission rate. \\
\hline$\alpha$ & $0.2 \mathrm{~dB} / \mathrm{km}$ & Fiber loss coefficient. \\
\hline$L_{\mathrm{Mx}}$ & $3 \mathrm{~dB}$ & Multiplexer insertion loss. \\
\hline$L_{\mathrm{Dx}}$ & $3 \mathrm{~dB}$ & Demultiplexer insertion loss. \\
\hline$L_{\mathrm{Sw}}$ & $3 \mathrm{~dB}$ & Switch Loss. \\
\hline$\epsilon$ & $-40 \mathrm{~dB}$ & Isolation between ports at the switch. \\
\hline$D_{P M D}$ & $0.01 \mathrm{ps} / \sqrt{\mathrm{km}}$ & PMD coefficient. \\
\hline$F_{0}(\mathrm{NF})$ & $3.162(5 \mathrm{~dB})$ & Amplifier noise factor (Noise figure). \\
\hline
\end{tabular}

We also implemented three other well known routing algorithms to compare to the distance based ACO and the proposed ACO. These algorithms are shortest path (SP), minimum hops (Hopcount) and Least Resistance Weight (LRW). Shortest path algorithm selects the route with minimum distance. Minimum hops selects the route with the minimum number of hops in the lightpath. The Least Resistance Weight uses the less congestioned route.

We performed all the tests in a computer with the following configuration: Intel Core 2 Quad Q6600 2.4 GHz, L2 Cache memory of $8 \mathrm{MB}$, RAM memory of $4 \mathrm{~GB}$, a SATA II Hard 


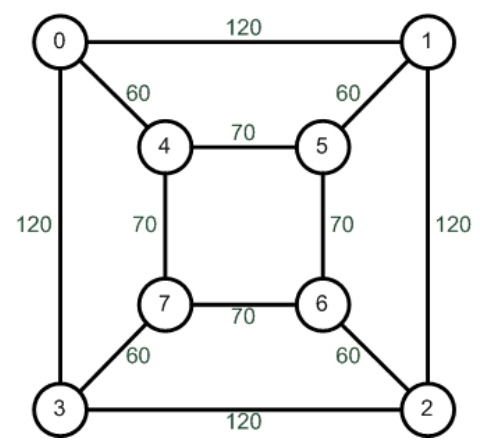

(a)

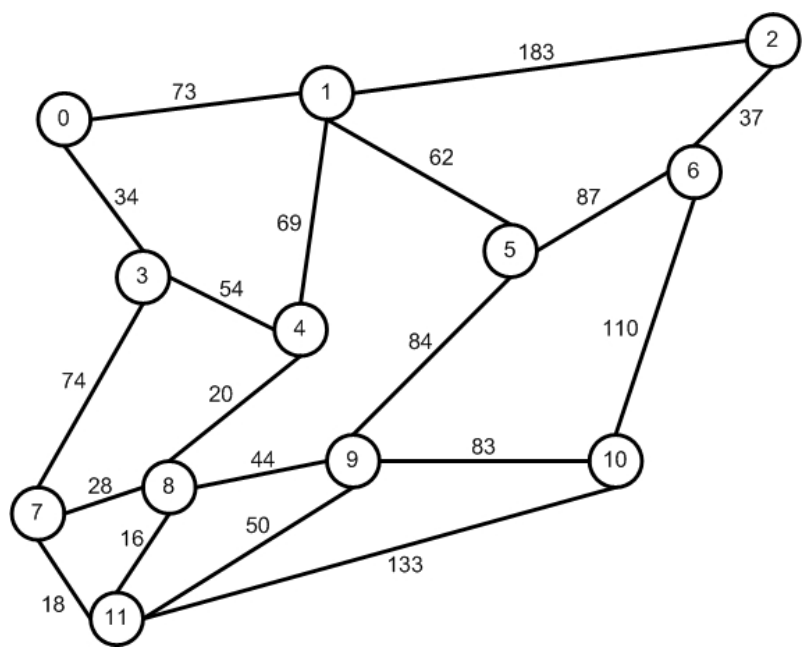

(b)

Fig. 1. Network topologies used in our simulations, regular (a) and Finland (b).

disk with storage capacity of 250 GB and 7.200 RPM. The time necessary to perform 150 PSO iterations was around three weeks and each point in the figures of the next section spent around 16 minutes to be evaluated.

\section{Simulation Results}

This section shows the results achieved for the proposed ACO RWA algorithm. We also present, for the sake of comparisson, the results of the distance based ACO, acording to the equation 1 . To obtain the function parameters of both approaches we performed independent training stages.

\section{A. Training Stage}

Considering the Regular topology, the trainning stage for the distance based ACO algorithm performed for 40 Erlangs and 16 wavelengths per link resulted in the PSO convergence around the $85^{t h}$ iteration, with blocking probability of approximately 0.013 . For the proposed ACO algorithm with 40 Erlangs and 16 wavelengths per link, the PSO converged earlier (around the $30^{\text {th }}$ iteration), reaching blocking probability around 0.009. For the distance based ACO with 85 Erlangs and 32 wavelengths per link the PSO convergence occurred in the second iteration of the simulation and the obtained blocking blocking probability was about 0.0026 . In the proposed ACO scenario with 85 Erlangs and 32 wavelengths per link, the
PSO converged after 15 iterations and the ACO RWA achieved blocking probabilities as low as 0.001 .

Table II shows the values of the cost function parameters obtained from the training phase simulations for the RWA based on ACO algorithms with the Regular network. In the scenario with 32 wavelengths, 85 Erlangs and the proposed ACO, three particles with different configurations had the same blocking probability values. Through performance test simulations with $10^{6}$ calls, it was confirmed that these combinations of weight values really generate similar results. The values $(\alpha=3.50144$, $\beta=3.01474, \gamma=3.48118$ ) were chosen for the performance evaluation of the proposed ACO routing algorithm on Regular network with 32 wavelengths per link, because the particle with this configuration was the first to reach the lowest call blocking probability during training phase.

TABLE II

VALUES OF COST FUNCTION PARAMETERS OBTAINED BY THE TRAINING PROCESS FOR RWA BASED ON ACO ALGORITHMS WITH THE REGULAR NETWORK.

\begin{tabular}{|c|c|c|l|}
\hline Regular Network Configuration & $\alpha$ & $\beta$ & $\gamma$ \\
\hline \hline $16 \lambda^{\prime}$ s, 40 Erl., ACO distance & 1.46175 & 1.92726 & - \\
\hline $16 \lambda^{\prime}$ s, 40 Erl., ACO Proposal & 2.08252 & 2.20869 & 2.96475 \\
\hline $32 \lambda^{\prime}$ s, 85 Erl., ACO distance & 2.67244 & 3.39465 & - \\
\hline $32 \lambda^{\prime}$ s, 85 Erl., ACO Proposal & 3.50144 & 3.01474 & 3.48118 \\
\hline $32 \lambda^{\prime}$ s, 85 Erl., ACO distance & 1.19588 & 1.56004 & 3.98344 \\
\hline $32 \lambda^{\prime}$ s, 85 Erl., ACO Proposal & 0.110748 & 1.99878 & 3.99688 \\
\hline
\end{tabular}

Considering the Finland topology, the trainning stage for the distance based ACO algorithm performed with 40 Erlangs and 16 wavelengths per link resulted in the PSO convergence around the $75^{\text {th }}$ iteration, with blocking probability of approximately 0.035 . For the proposed ACO function with 40 Erlangs and 16 wavelengths per link, the PSO converged earlier (around the $60^{\text {th }}$ iteration), reaching blocking probability around 0.028. For the distance based ACO with 90 Erlangs and 32 wavelengths per link the PSO convergence occured only in the $80^{t h}$ iteration of this simulation with minimum blocking probability of around 0.036 . For the proposed ACO function with 90 Erlangs and 32 wavelengths per link, the PSO converged after 75 iterations and the ACO RWA achieved blocking probabilities of 0.031 .

Table III shows the values for the weight function parameters obtained by the training stage for RWA based on ACO algorithms with the Finland network. In both scenarios, the most relevant parameter for distance based ACO was pheromone accumulation, and for the proposed ACO algorithm was the wavelength availability for 16 wavelengths and the pheromone accumulation for 32 wavelengths. Note that the importance of the coefficients changed according to network conditions.

\section{B. Performance Analysis}

We tested and compared the performance of the RWA algorithms on four scenarios, changing network topology, load and wavelength availability per link. Figure 2(a) and Figure 2(b) present the network blocking probability as a function of network load, for five different RWA algorithms, 
TABLE III

INDUCTION EQUATION WEIGHT VALUES OBTAINED BY THE TRAINING PROCESS FOR RWA BASED ON ACO ALGORITHMS WITH THE FINLAND NETWORK.

\begin{tabular}{|c|c|c|l|}
\hline Regular Network Configuration & $\alpha$ & $\beta$ & $\gamma$ \\
\hline \hline $16 \lambda^{\prime}$ s, 40 Erl., ACO distance & 0.398342 & 0.00284 & - \\
\hline $16 \lambda^{\prime}$ s, 40 Erl., ACO Proposal & 0.729141 & 1.32989 & 3.33693 \\
\hline $32 \lambda^{\prime}$ s, 90 Erl., ACO distance & 3.97025 & 2.80346 & - \\
\hline $32 \lambda^{\prime}$ s, 90 Erl., ACO Proposal & 4.46353 & 0.70601 & 0.10476 \\
\hline
\end{tabular}

obtained on the Regular topology with 16 wavelengths and 32 wavelengths, respectively. The values shown are the average values of the total blocking probabilities, obtained from five simulation runs.

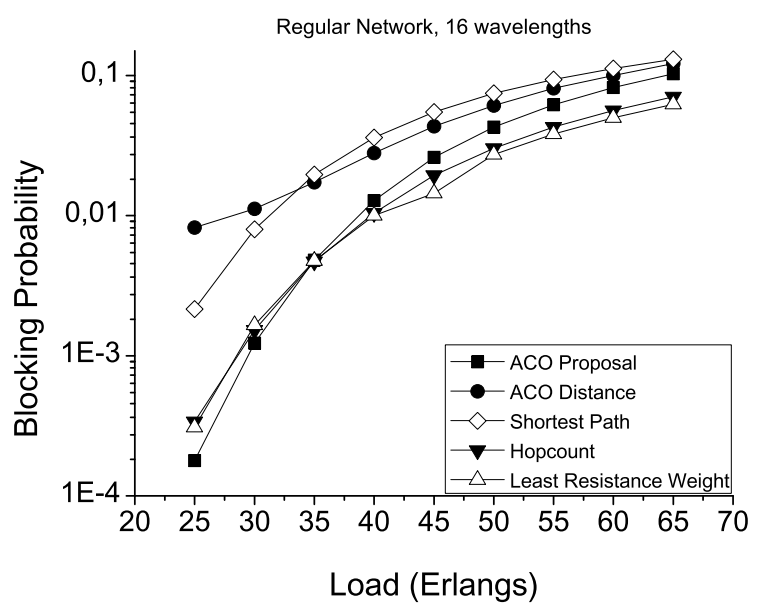

(a)

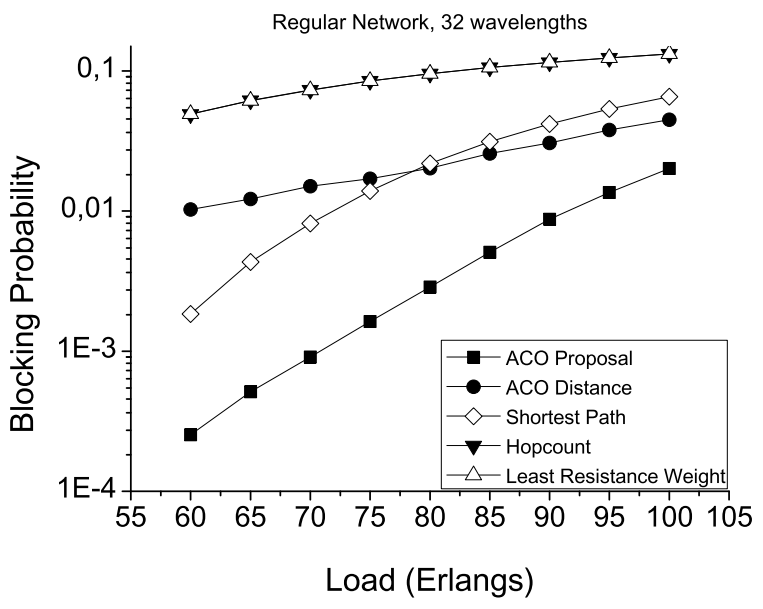

(b)

Fig. 2. Performance comparison of the RWA algorithms. Regular network links with (a) 16 wavelengths, and (b) 32 wavelengths.

Figure 2(a) shows that the proposed ACO achieved the best performance for loads of 25 and 30 Erlangs. When the load was increased to 35 Erlangs and beyond, the RWA algorithms based on hopcount, and load balancing presented slightly better performance than the proposed ACO. However, for any network load our proposed ACO algorithm performed better than the ACO based on distance or the shortest path algorithms. These results show that taking into account the wavelength availability in the weight function leads to an improved performance for the proposed ACO, compared to the ACO algorithm based on distance.

Figure 2(b) shows similar results to Figure 2(a), but with 32 wavelengths per link. In this scenario there is no call blocking due to lack of available wavelength. Instead, the call blockings are due to the physical impairments. In this scenario the proposed ACO algorithm outperformed by a large margin the other algorithms. This result indicates that it is worth finding the ideal balance between pheromone, link length and wavelength availability in the ACO cost function, through the optimization of the $\alpha, \beta$ and $\gamma$ parameters.

We analysed the boxplot graphics for the ACO based on distance and for the proposed ACO IA-RWA in the regular topology, for 16 and 32 wavelengths. The highest value of the standard deviation obtained in the simulations was 0.00158 , for 60 Erlangs and 16 wavelengths. The largest percentage change between the lower and upper limits was $23.49 \%$, for 25 Erlangs and 16 wavelengths.

Figure 3(a) and Figure 3(b) shows the blocking probability as a function of network load for different RWA algorithms, obtained from Finland network with 16 wavelengths and 32 wavelengths, respectively. These figures show that the proposed ACO, the ACO based on distance and the shortest path algorithms presented similar performance, which is considerably better than the hopcount and LRW algorithms

We analysed the boxplot graphics for the ACO based on distance and the proposed ACO IA-RWA in the Finland topology, for 16 and 32 wavelengths. The standard deviation obtained in simulations with ACO Proposal was 0.00187 , for 55 Erlangs and 32 wavelengths. The largest percentage change between the lower and upper limits was $9.54 \%$, for 15 Erlangs and 16 wavelengths for ACO proposed.

One can note by observing the simulation results that our approach did not far outperform the other approaches presented for comparison in all the cases. However, the algorithm features an adaptable behaviour. It means that in some cases the Least Resistance Weight algorithm outperformed the Hop Count algorithm, while the Shortest Path algorithm outperformed the Least Resistance Weight algorithm in other cases. However, our proposal presented good results in all the cases.

\section{CONCLUSIONS}

In this paper we proposed and analyzed the performance of an IA-RWA for all-optical networks that includes both the physical distance and the wavelength availability based on Ant Colony Optimization technique and trained with Particle Swarm Optimization to obtain the optimized parameters for the ACO cost function. We analyzed two approaches for ACO based RWA algorithm, one that considers pheromone quantity and link distance on the cost function and another one that also includes wavelength availability in the cost function. We compared the performance of both approaches to classical RWA algorithms in terms of blocking probability. 


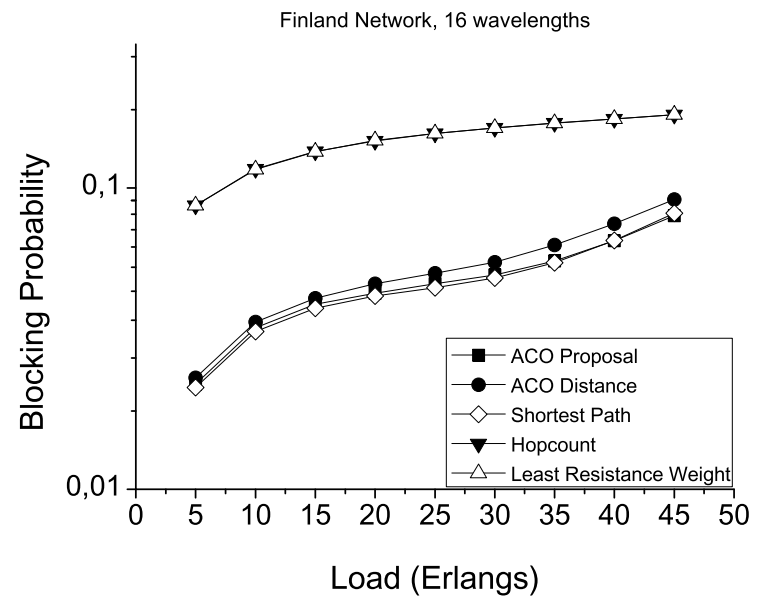

(a)

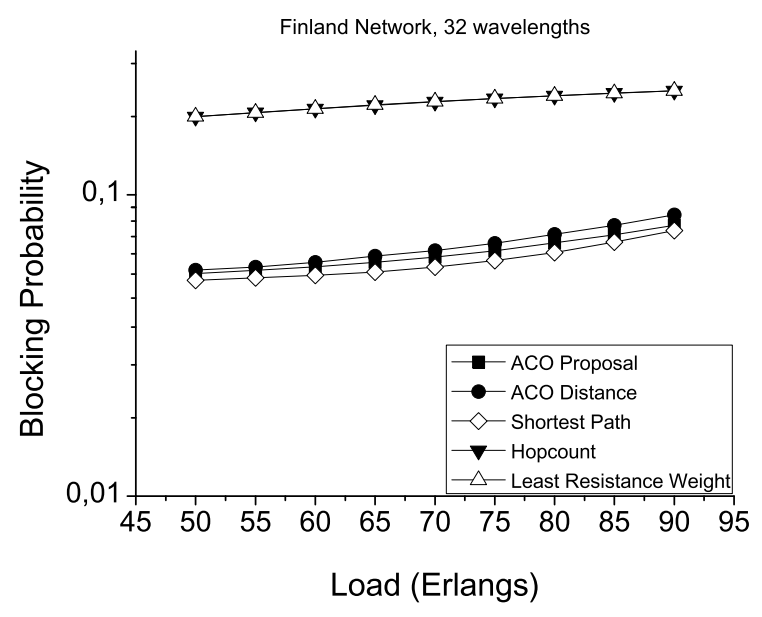

(b)

Fig. 3. Performance comparison of the RWA algorithms. Finland network links with (a) 16 wavelengths, and (b) 32 wavelengths.

The results for different network topologies and conditions shown that our proposed ACO IA-RWA algorithm has the ability to adapt itself to the different conditions and perform better or at least equal to the ACO based on distance and the classical RWA algorithms.

One should note that other approaches such as the one proposed in [11] can be combined with our approach. Furthermore, the heuristic based on the link wavelength availability of the network links presented in [13] is similar to our proposal when $\beta=0$ and $\alpha=\gamma=1$.

As we perform a training stage to find the best set of parameters $(\alpha, \beta$ and $\gamma)$, we believe that this novel heuristic can include other physical layer aspects in the routing process. As can be seen from equation 3, the percentage of available wavelengths $\left(\Delta \lambda_{i j}\right)$ multiplies the previous equation and is weighted by $\gamma$. We think that the novel equation presented in 3 with the trainning stage is our major contribution.

\section{REFERENCES}

[1] B. Mukherjee, "WDM Optical Communication Networks: Progress and Challenges," Journal of Selected Areas in Communications, v. 18, n. 10, pp. 1810-1824, 2000.
[2] P. Green, "Progress in Optical Networking," IEEE Communications Magazine, v. 39, n. 1, pp. 54-61, 2001.

[3] Z. Zhang, J. Fu, D. Guo and L. Zhang, "Lightpath routing for intelligent optical networks," IEEE Network, v. 15, n. 4, pp. 28-35, 2001.

[4] R. Ramaswami and K. N. Sivarajan, "Routing and wavelength assignment in all-optical networks," IEEE/ACM Transactions on Networking, v. 3, n. 5, pp. 489-500, 1995.

[5] J. Strand, A. L. Chiu and R. Tkach, "Issues for routing in the optical layer," IEEE Communications Magazine, v. 39, n. 2, pp. 81-87, 2001.

[6] C. J. A. Bastos-Filho, W. H. Schuler, A. L. I. Oliveira, "Routing algorithm based on Swarm Intelligence and Hopfield Neural Network applied to communication networks," Electronics Letters, v. 44, pp. 4445, 2008.

[7] D. Bisbal et al., "Dynamic Routing and Wavelength Assignment in Optical Networks by Means of Genetic Algorithms," Photonic Network Communications, v. 7, n. 1, pp. 43-58, 2004.

[8] J. F. Martins-Filho, D. A. R. Chaves, C. J. A. Bastos-Filho, D. O. Aguiar, "Intelligent and fast IRWA algorithm based on power series and particle swarm optimization," International Conference on Transparent Optical Networks, v. 3, Athens, Greece, pp. 158-161, 2008.

[9] G. Di Caro and M. Dorigo, "AntNet: Distributed Stigmergetic Control for Communications Networks," Journal of Artificial Intelligence Research, v. 9, pp. 317-365, 1998.

[10] S.-H. Ngo, X. Jiang and S. Horiguchi, "Adaptive routing and wavelength assignment using ant-based algorithm," 12th IEEE International Conference on Networks, v. 2, pp. 482-486, 2004.

[11] G. S. Pavani and H. Waldman, "Adaptive Routing and Wavelength Assignment with Power Constraints using Ant Colony Optimization," VI International Telecommunications Symposium, n. 10, pp. 507-512, Fortaleza/CE, Brazil, 2006

[12] G. S. Pavani, L. G. Zuliani, H. Waldman and M. Magalhaes, "Distributed approaches for impairment-aware routing and wavelength assignment algorithms in GMPLS networks," Computer Networks, v. 52, n. 10, pp. 1905-1915, 2008.

[13] G. S. Pavani and H. Waldman, "Restoration in wavelength-routed optical networks by means of ant colony optimization," Photonic Network Communications, v. 16, n. 1, pp. 83-91, 2008.

[14] K. M. Sim and W. H. Sun, "Ant colony optimization for routing and load-balancing: survey and new directions," IEEE Transactions on Systems, Man, and Cybernetics, Part A, v. 33, n. 5, pp. 560-572, 2003.

[15] D. Bratton and J. Kennedy, "Defining a Standard for Particle Swarm Optimization," Swarm Intelligence Symposium, 2007. SIS 2007. IEEE, pp. 120-127, Honolulu, USA, 2007.

[16] M. Clerc and J. Kennedy, "The particle swarm - explosion, stability, and convergence in a multidimensional complex space," IEEE Transactions on Evolutionary Computation, v. 6, n. 1, pp. 58-73, 2002.

[17] D. F. Carvalho, "Roteamento em Redes Ópticas Transparentes Utilizando Otimização por Colônias de Formigas" M.Sc. Dissertation, University of Pernambuco, 118 pages, 2008.

[18] H. A. Pereira, D. A. R. Chaves, C. J. A. Bastos-Filho, and J. F. Martins-Filho, "OSNR model to consider physical layer impairments in transparent optical networks," Photonic Network Communications, Springer, Online 2008, http://dx.doi.org/10.1007/s11107-008-0178-2. 